\title{
Genetic determinants of freckle occurrence in the Spanish population: Towards ephelides prediction from human DNA samples
}

\section{Barbara Hernando}

Maria Victoria Ibañez ${ }^{\mathrm{b}}$

Julio Alberto Deserio-Cuesta ${ }^{\mathrm{a}}$

Raquel Soria-Navarro ${ }^{\mathrm{a}}$

Inca Vilar-Sastre ${ }^{\mathrm{a}}$

\section{Conrado Martínez-Cadenas ${ }^{\mathrm{a},}$}

ccadenas@uji.es

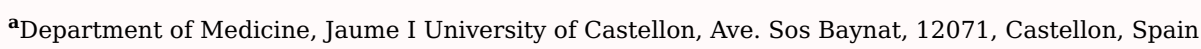

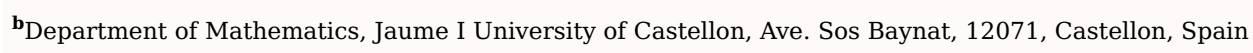

${ }^{*}$ Corresponding author.

\section{Abstract}

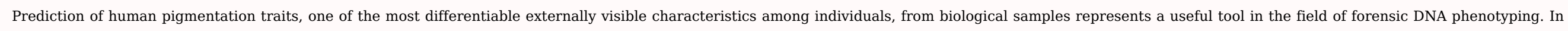

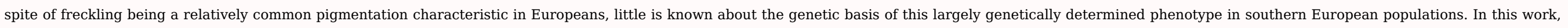

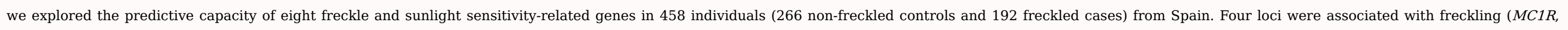

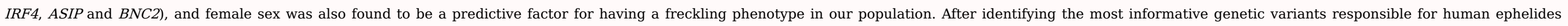

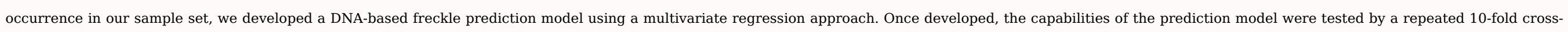

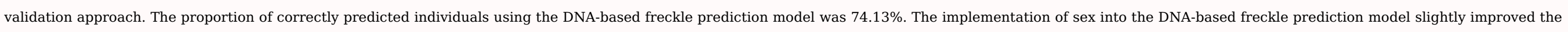

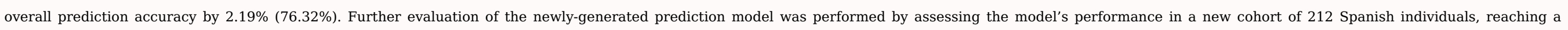

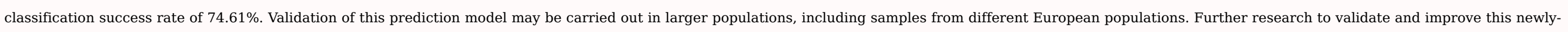

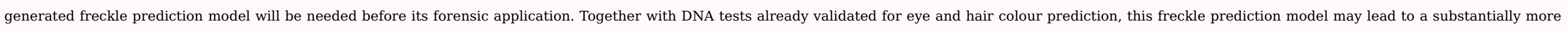
detailed physical description of unknown individuals from DNA found at the crime scene.

Keywords: Freckles; Ephelides; Externally visible traits; DNA-based prediction; Forensic science

\section{Introduction}

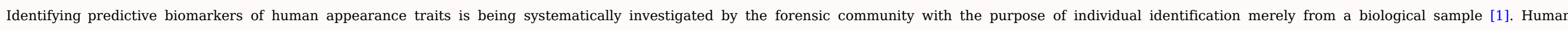

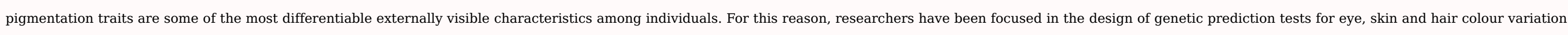
[2-7]. However, DNA-based prediction of other human pigmentation traits under a strong genetic control, such as ephelides occurrence, has not been generated yet.

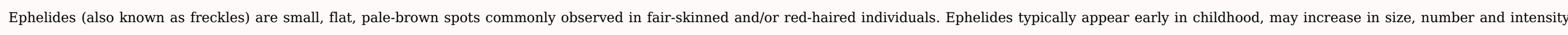

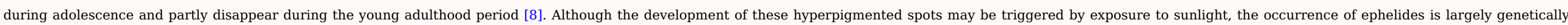




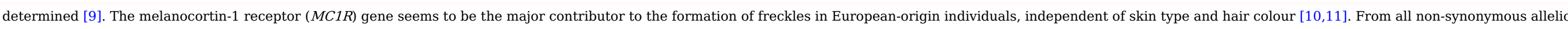

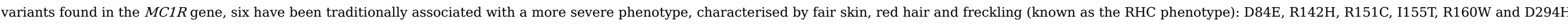

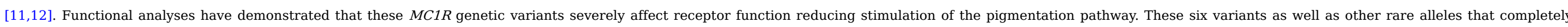

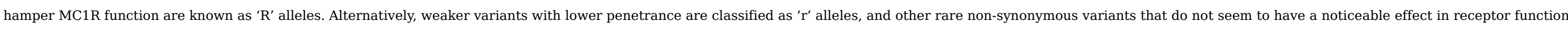
are defined as pseudoalleles [13].

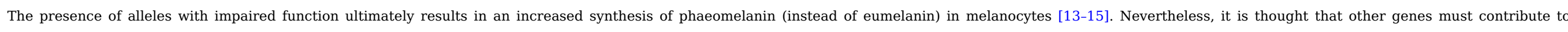

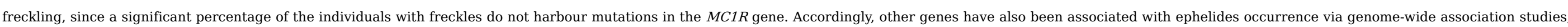
including IRF4, ASIP, TYR and BNC2 [16-18].

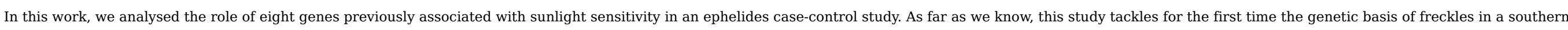

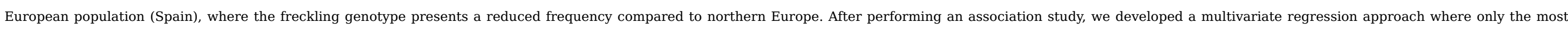

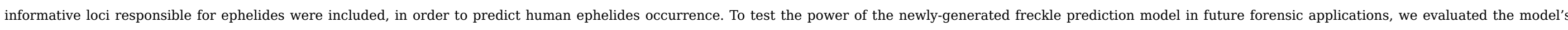
prediction performance in terms of accuracy, sensitivity and specificity by means of a cross-validation approach as well as an external validation using an independent sample.

\section{Materials and methods}

\subsection{Study subjects and data collection}

\subsubsection{Original population}

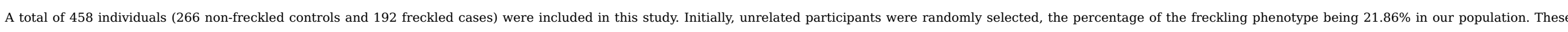

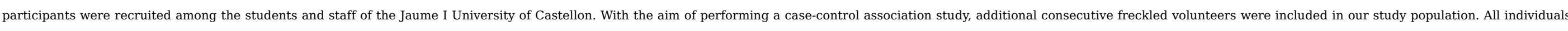
were Europeans of Spanish origin. Written informed consent was provided by all participants, and the study was approved by the Ethics Committee of the Jaume I University of Castellon (Castellon, Spain)

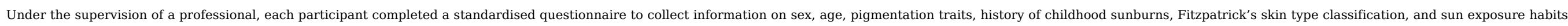

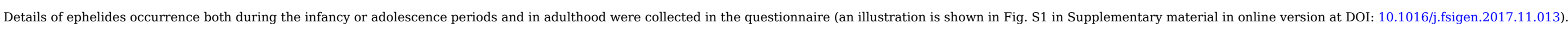

\subsubsection{Independent validation population}

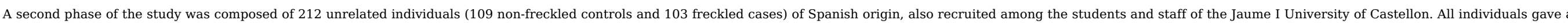
written informed consent and completed the standardised questionnaire used to collect phenotypic information

\subsection{DNA extraction}

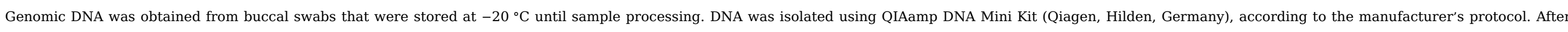
DNA extraction, all samples were diluted to a concentration of $5 \mathrm{ng} / \mathrm{\mu l}$ in order to prepare them for DNAPCR amplification.

\subsection{SNP selection and genotyping}

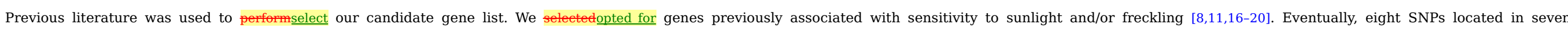

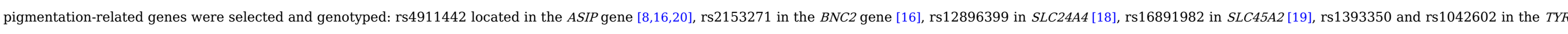

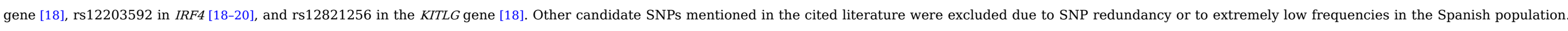
SNP codes, locations, ancestral and derived alleles and their frequencies were obtained from the Ensembl Variation database (http://www.ensembl.org/info/genome/variation/index.html).

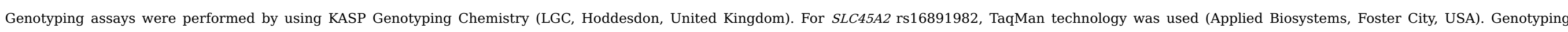

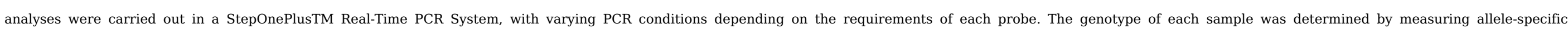




\subsection{Sequencing of $M C 1 R$ coding region}

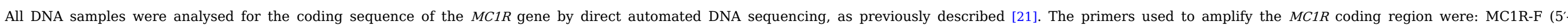

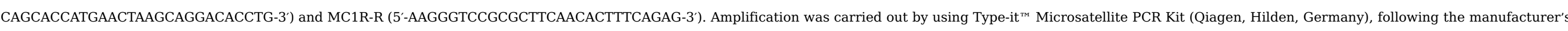

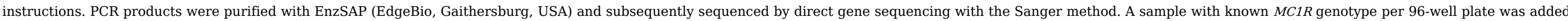
for quality control. All sequencing results were analysed using SeqScape v2.5 software to align and detect all nucleotide changes. All detected sequence changes were confirmed manually.

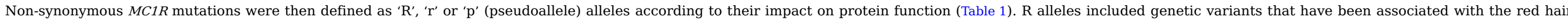

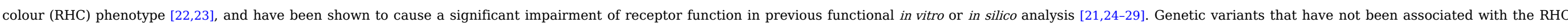

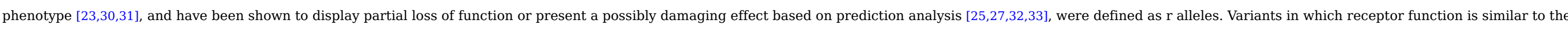
wild-type form were catalogued as $\mathrm{p}$ alleles. Individuals were classified based on the number of $\mathrm{R}$ and/or $\mathrm{r}$ alleles carried.

Table $1 M C 1 R$ variants with protein sequence alterations identified in the current study.

\begin{tabular}{|c|c|c|c|c|c|}
\hline Variant & Nucleotide change & Functional analysis & Prediction analysis & Polyphen score & Reference \\
\hline \multicolumn{6}{|l|}{$\mathrm{R}$ alleles } \\
\hline D84E & c. $252 \mathrm{C}>\mathrm{A}$ & Yes & - & - & Beaumont et al. [23] \\
\hline $\mathrm{R} 142 \mathrm{H}$ & c. $425 \mathrm{G}>\mathrm{A}$ & Yes & - & - & Beaumont et al. [23] \\
\hline R151C & c. $451 \mathrm{C}>\mathrm{T}$ & Yes & - & - & Beaumont et al. [22] \\
\hline I155T & c. $464 \mathrm{~T}>\mathrm{C}$ & Yes & - & - & Beaumont et al. [23] \\
\hline R160W & c. $478 \mathrm{C}>\mathrm{T}$ & Yes & - & - & Beaumont et al. [22] \\
\hline D294H & c. $880 \mathrm{G}>\mathrm{C}$ & Yes & - & - & Beaumont et al. (2006)[22] \\
\hline Q30X & c. $88 \mathrm{C}>\mathrm{T}$ & No & No & - & Guan et al. [24] \\
\hline $\mathrm{C} 35 \mathrm{Y}$ & c. $104 \mathrm{G}>\mathrm{A}$ & Yes & - & - & Fargnoli et al. [32] and Fernandez et al. [29] \\
\hline S41F & c. $122 \mathrm{C}>\mathrm{T}$ & Yes & - & - & Pérez Oliva et al. [28] \\
\hline S83P & c. $247 \mathrm{~T}>\mathrm{C}$ & No & Yes & - & Kanetsky et al. [26] and Ibarrola-Villava et al. (2013)[36] \\
\hline S83L & c. $248 \mathrm{C}>\mathrm{T}$ & Yes & - & - & Ozola et al. [27] \\
\hline G89R & c. $265 \mathrm{G}>\mathrm{C}$ & Yes & - & - & Ozola et al. [27] \\
\hline M128T & c. $383 \mathrm{~T}>\mathrm{C}$ & Yes & - & - & Pérez Oliva et al. [28] \\
\hline L135R & c. $404 \mathrm{~T}>\mathrm{G}$ & No & No & 1.000 & NEW. Never reported/not found in SNPs databases \\
\hline Y152X & c. $456 \mathrm{C}>\mathrm{A}$ & Yes & - & - & Fargnoli et al. [32] \\
\hline Q233X & c. $697 \mathrm{C}>\mathrm{T}$ & No & No & - & Martinez-Cadenas et al. [35] \\
\hline P256S & c. $766 \mathrm{C}>\mathrm{T}$ & No & Yes & - & Hu et al. (2014) Kanetsky et al. [26] \\
\hline
\end{tabular}




\begin{tabular}{|c|c|c|c|c|c|}
\hline P268R & c. $803 \mathrm{C}>\mathrm{G}$ & No & Yes & - & Ibarrola-Villava et al. [25] \\
\hline \multicolumn{6}{|l|}{$\mathrm{r}$ alleles } \\
\hline V60L & c. $178 \mathrm{G}>\mathrm{T}$ & Yes & - & - & Beaumont et al. [23] and Herraiz et al. [30] \\
\hline V92M & c. $274 \mathrm{G}>\mathrm{A}$ & Yes & - & - & Beaumont et al. [23] and Herraiz et al. [30] \\
\hline R163Q & c. $488 \mathrm{G}>\mathrm{A}$ & Yes & - & - & Beaumont et al. [23] and Nakayama et al. [31] \\
\hline $\mathrm{L} 24 \mathrm{M}$ & c. $70 \mathrm{C}>\mathrm{A}$ & No & No & 0.863 & NECTAR (http://nectarmutation.org) \\
\hline F45L & c. $133 \mathrm{~T}>\mathrm{C}$ & No & Yes & - & Ozola et al. [27] and Ibarrola-Villava et al. [25] \\
\hline L46V & c. $136 \mathrm{C}>\mathrm{G}$ & No & No & 0.741 & Ensembl (www.ensembl.org) \\
\hline R67Q & c. $200 \mathrm{G}>\mathrm{A}$ & No & Yes & - & Fargnoli et al. [32] \\
\hline T95M & c. $284 \mathrm{C}>\mathrm{T}$ & Yes & - & - & Ozola et al. [27] \\
\hline I120T & c.359T > C & No & Yes & - & Fargnoli et al. [32] \\
\hline V122M & c. $364 \mathrm{G}>\mathrm{A}$ & Yes & - & - & Jimenez-Cervantes et al. [33] \\
\hline V156A & c. $467 \mathrm{~T}>\mathrm{C}$ & No & No & 0.784 & Kanetsky et al. [26] \\
\hline V193L & c. $577 \mathrm{G}>\mathrm{T}$ & No & No & 0.567 & Ensembl (www.ensembl.org) \\
\hline N279K & c. $837 \mathrm{C}>\mathrm{A}$ & No & Yes & - & Fargnoli et al. [32] and Ibarrola-Villava et al. [25] \\
\hline \multicolumn{6}{|l|}{$\mathrm{p}$ alleles } \\
\hline G32R & c. $94 \mathrm{G}>\mathrm{A}$ & No & No & 0.299 & NEW. Never reported/not found in SNPs databases \\
\hline S47T & c. $140 \mathrm{G}>\mathrm{C}$ & No & No & 0.003 & Gan-Or et al. (2016)Garcia-Borron et al. [57] \\
\hline A57V & c. $170 \mathrm{C}>\mathrm{T}$ & No & No & 0.001 & Ensembl (www.ensembl.org) \\
\hline G89E & c. $265 \mathrm{G}>\mathrm{A}$ & No & No & 0.170 & Ensembl (www.ensembl.org) \\
\hline M128V & c. $382 \mathrm{~A}>\mathrm{G}$ & No & No & 0.019 & NECTAR (http://nectarmutation.org) \\
\hline A167T & c. $499 \mathrm{G}>\mathrm{A}$ & No & No & 0.024 & Ensembl (www.ensembl.org) \\
\hline V208I & c. $622 \mathrm{G}>\mathrm{A}$ & No & No & 0.002 & Ensembl (www.ensembl.org) \\
\hline
\end{tabular}

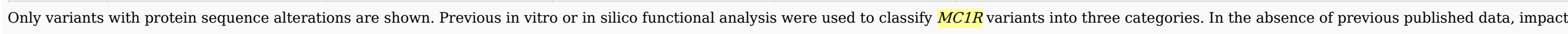

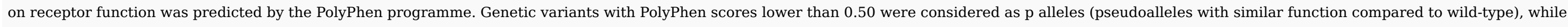
variants with scores from 0.50 to 0.95 were classified as $r$ alleles (possibly damaging).

\subsection{Association analysis}

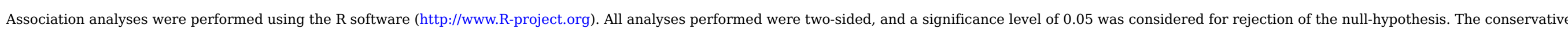
Bonferroni correction was used to adjust the significance level for multiple testing ( $P$-value $<4.54 \times 10^{-3}=0.05 / 11$ ). Unknown and missing values were excluded at each specific analysis.

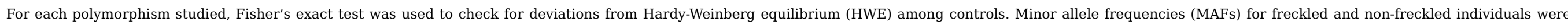
estimated from our population. 


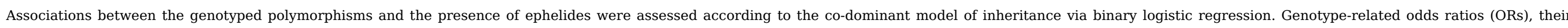

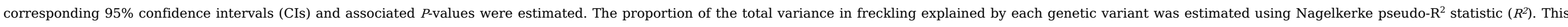
statistic parameter was used to rank the genetic variants included in the study based on their contribution to the freckling phenotype.

\subsection{Prediction model}

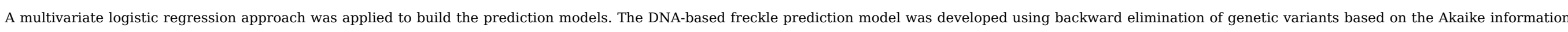

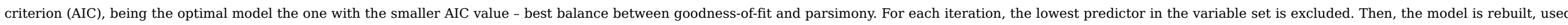

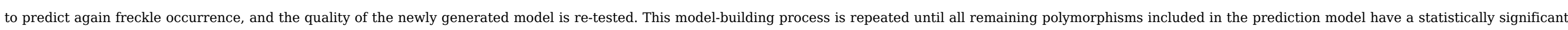
contribution, and the estimated information loss is minimised.

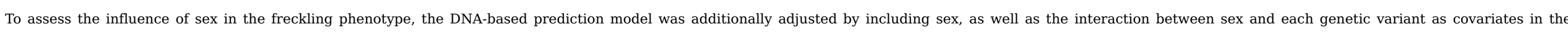
multivariate logistic regression. As above, the optimal sex-adjusted prediction model was developed based on the AIC variable selection criteria.

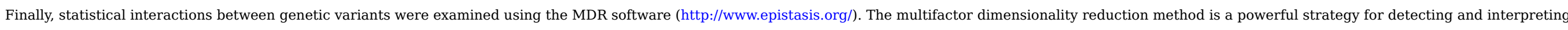

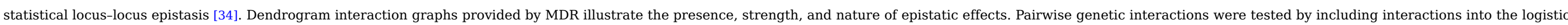
regression model.

\subsection{Model evaluation}

\subsubsection{Internal evaluation phase - cross-validation}

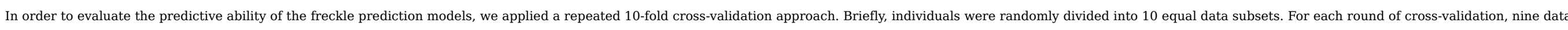

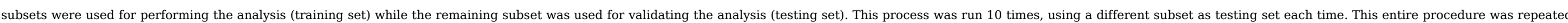

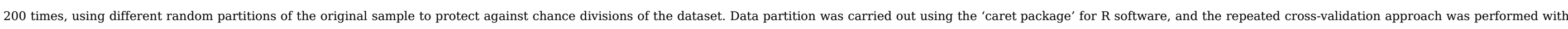
custom programmes written in $\mathrm{R}$.

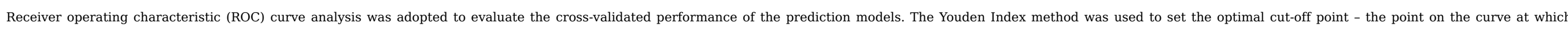

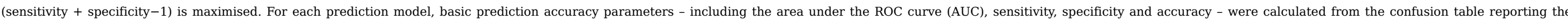

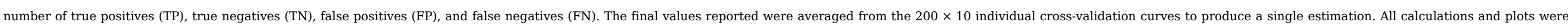
performed using the 'ROCR package' for R software.

\subsubsection{External evaluation phase - independent population}

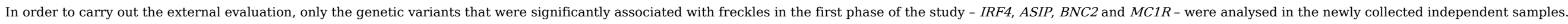

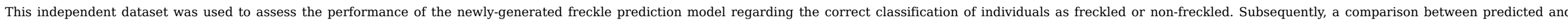
observed data was performed, and the basic accuracy parameters (AUC, sensitivity, specificity and accuracy) were calculated from the confusion table by using the 'pROC package' for R software.

\section{Results}

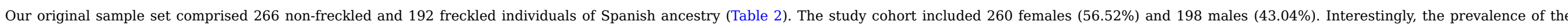

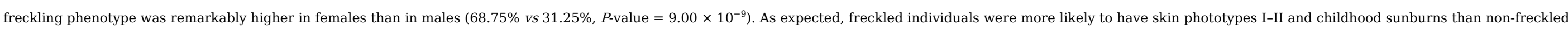
individuals, although no significant differences between them were found regarding sun exposure habits (Table 2).

Table 2 Classification of the Spanish individuals according to sex, skin phototype and sun exposure habits. 


\begin{tabular}{|c|c|c|c|c|c|}
\hline Female & 128 & 48.12 & 132 & 68.75 & 9.00E-06 \\
\hline Male & 138 & 51.88 & 60 & 31.25 & \\
\hline Unknown & 0 & 0.00 & 0 & 0.00 & \\
\hline \multicolumn{6}{|l|}{ Sun exposure } \\
\hline Never & 22 & 8.27 & 17 & 8.85 & 0.438 \\
\hline Intermittent & 134 & 50.38 & 106 & 55.21 & \\
\hline Habitual & 109 & 40.98 & 65 & 33.85 & \\
\hline Unknown & 1 & 0.38 & 9 & 4.69 & \\
\hline \multicolumn{6}{|c|}{ Skin Phototype } \\
\hline III/IV & 203 & 76.32 & 69 & 35.94 & 9.20E-18 \\
\hline I/II & 63 & 23.68 & 121 & 63.02 & \\
\hline Unknown & 0 & 0.00 & 2 & 1.04 & \\
\hline \multicolumn{6}{|c|}{ Childhood sunburns } \\
\hline No & 126 & 47.37 & 37 & 19.27 & 3.52E-09 \\
\hline Yes & 138 & 51.88 & 145 & 75.52 & \\
\hline Unknown & 2 & 0.75 & 10 & 5.21 & \\
\hline
\end{tabular}

$\mathrm{N}$, number of individuals; \%, percentage of individuals per group among the total.

a $P$-value for the Fisher's exact test. Significant results are presented in bold.

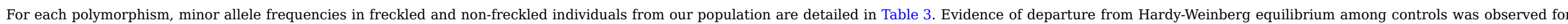

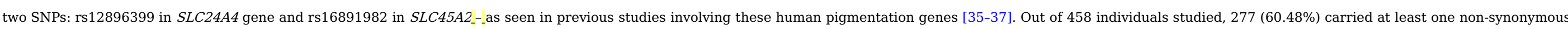

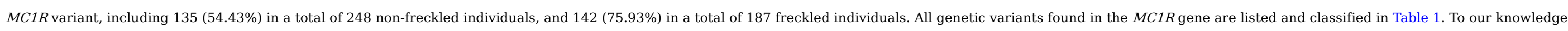

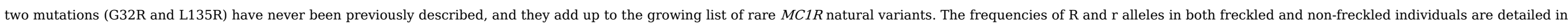
Table 3. Seventeen samples were discarded due to unsuccessful $M C 1 R$ sequencing.

Table 3 Genotypic association with freckles in the Spanish population.

alt-text: Table 3

Gene

SNP \# rs No. Genotype

Non--_freckled individuals

Freckled individuals

$P$-value ${ }^{\mathrm{a}}$

OR (95\% CI)

$R^{2}(\%)^{\mathrm{b}}$ 


\begin{tabular}{|c|c|c|c|c|c|c|c|c|c|c|c|c|c|}
\hline & & & $\mathrm{N}$ & $\%$ & MAF & HWE $P$-value & $\mathrm{N}$ & $\%$ & MAF & & & & \\
\hline \multirow[t]{4}{*}{$A S I P$} & rs4911442 & $\mathrm{AA}$ & 233 & 87.59 & 0.062 & 0.264 & 153 & 79.69 & 0.109 & 0.044 & reference & 1.01 & 4 \\
\hline & & AG & 29 & 10.90 & & & 36 & 18.75 & & & $1.89(1.11-3.21)$ & & \\
\hline & & GG & 2 & 0.75 & & & 3 & 1.56 & & & $2.28(0.38-13.83)$ & & \\
\hline & & ND & 2 & 0.75 & & & 0 & 0.00 & & & & & \\
\hline \multirow[t]{4}{*}{$B N C 2$} & rs2153271 & TT & 68 & 25.56 & 0.476 & 0.222 & 61 & 31.77 & 0.406 & 0.067 & reference & 0.87 & 5 \\
\hline & & $\mathrm{CT}$ & 143 & 53.76 & & & 106 & 55.21 & & & $0.83(0.54-1.27)$ & & \\
\hline & & $\mathrm{CC}$ & 55 & 20.68 & & & 25 & 13.02 & & & $0.51(0.28-0.91)$ & & \\
\hline & & ND & 0 & 0.00 & & & 0 & 0.00 & & & & & \\
\hline \multirow[t]{4}{*}{ IRF4 } & rs12203592 & $\mathrm{CC}$ & 207 & 77.82 & 0.117 & 1.000 & 100 & 52.08 & 0.275 & $1.81 E-05^{*}$ & reference & 5.92 & 2 \\
\hline & & $\mathrm{CT}$ & 56 & 21.05 & & & 77 & 40.10 & & & $2.49(1.57-3.94)$ & & \\
\hline & & TT & 3 & 1.13 & & & 14 & 7.29 & & & $6.56(1.68-25.54)$ & & \\
\hline & & ND & 0 & 0.00 & & & 1 & 0.52 & & & & & \\
\hline \multirow[t]{4}{*}{ KITLG } & rs12821256 & TT & 243 & 91.35 & 0.041 & 1.000 & 175 & 91.15 & 0.034 & 0.417 & reference & 0.11 & 9 \\
\hline & & $\mathrm{CT}$ & 21 & 7.89 & & & 11 & 5.73 & & & $0.73(0.34-1.56)$ & & \\
\hline & & $\mathrm{CC}$ & 0 & 0.00 & & & 0 & 0.00 & & & - & & \\
\hline & & ND & 2 & 0.75 & & & 6 & 3.13 & & & & & \\
\hline \multirow[t]{4}{*}{$M C 1 R$} & $\mathrm{R}$ alleles & $0 / 0$ & 214 & 80.45 & - & - & 110 & 57.29 & - & $7.05 E-08^{*}$ & reference & 6.91 & 1 \\
\hline & & $0 / 1$ & 38 & 14.29 & & & 64 & 33.33 & & & $3.26(2.05-5.18)$ & & \\
\hline & & $1 / 1$ & 2 & 0.75 & & & 13 & 6.77 & & & $11.61(2.55-52.83)$ & & \\
\hline & & ND & 12 & 4.51 & & & 5 & 2.60 & & & & & \\
\hline \multirow[t]{4}{*}{$M C 1 R$} & $r$ alleles & $0 / 0$ & 154 & 57.89 & - & - & 92 & 47.92 & - & 0.039 & reference & 1.09 & 3 \\
\hline & & $0 / 1$ & 86 & 32.33 & & & 77 & 40.10 & & & $1.49(1.01-2.24)$ & & \\
\hline & & $1 / 1$ & 14 & 5.26 & & & 18 & 9.38 & & & $2.15(1.02-4.53)$ & & \\
\hline & & ND & 12 & 4.51 & & & 5 & 2.60 & & & & & \\
\hline \multirow[t]{4}{*}{$S L C 24 A 4$} & rs12896399 & TT & 92 & 34.59 & 0.439 & 0.033 & 58 & 30.21 & 0.479 & 0.521 & reference & 0.21 & 7 \\
\hline & & GT & 113 & 42.48 & & & 85 & 44.27 & & & $1.20(0.77-1.85)$ & & \\
\hline & & GG & 60 & 22.56 & & & 49 & 25.52 & & & $1.33(0.80-2.19)$ & & \\
\hline & & ND & 1 & 0.38 & & & 0 & 0.00 & & & & & \\
\hline \multirow[t]{3}{*}{$S L C 45 A 2$} & rs16891982 & GG & 209 & 78.57 & 0.133 & 0.002 & 154 & 80.21 & 0.130 & 0.690 & reference & 0.12 & 8 \\
\hline & & CG & 43 & 16.17 & & & 26 & 13.54 & & & $0.82(0.48-1.39)$ & & \\
\hline & & $\mathrm{CC}$ & 14 & 5.26 & & & 12 & 6.25 & & & $1.16(0.52-2.59)$ & & \\
\hline
\end{tabular}




\begin{tabular}{|c|c|c|c|c|c|c|c|c|c|c|c|c|c|}
\hline & & ND & 0 & 0.00 & & & 0 & 0.00 & & & & & \\
\hline \multirow[t]{4}{*}{$T Y R$} & rs1393350 & GG & 75 & 28.30 & 0.239 & 1.000 & 58 & 30.21 & 0.259 & 0.777 & reference & 0.08 & 10 \\
\hline & & AG & 134 & 50.57 & & & 102 & 53.13 & & & $1.10(0.75-1.63)$ & & \\
\hline & & $\mathrm{AA}$ & 56 & 21.13 & & & 32 & 16.67 & & & $1.27(0.58-2.78)$ & & \\
\hline & & ND & 1 & 0.38 & & & 0 & 0.00 & & & & & \\
\hline \multirow[t]{4}{*}{$T Y R$} & rs1042602 & $\mathrm{CC}$ & 154 & 57.89 & 0.464 & 0.902 & 105 & 54.97 & 0.432 & 0.485 & reference & 0.23 & 6 \\
\hline & & $\mathrm{AC}$ & 97 & 36.47 & & & 73 & 38.22 & & & $0.98(0.64-1.51)$ & & \\
\hline & & $\mathrm{AA}$ & 15 & 5.64 & & & 13 & 6.81 & & & $0.74(0.42-1.28)$ & & \\
\hline & & ND & 0 & 0.00 & & & 1 & 0.52 & & & & & \\
\hline
\end{tabular}

SNP, single nucleotide polymorphism; N, number of individuals; \%, percentage of individuals per group among the total; OR, odds ratio per minor allele; CI, confidence interval; $R^{2}$, Nagelkerke pseudo- $\mathrm{R}^{2}$ statistic.

${ }^{*}$ Indicates significant results at Bonferroni threshold of 4.50E-03.

a $P$-value for the binary logistic regression association analysis according to the co-dominant model of inheritance. Bold indicates statistically significant results.

${ }^{\mathbf{b}}$ The proportion of total variance in freckling phenotype explained by the genetic variants was estimated using $R^{2}$.

${ }^{\mathbf{c}}$ The genetic variants were ranked according to their importance in freckling phenotype.

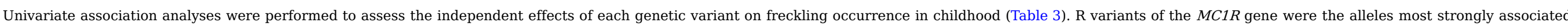

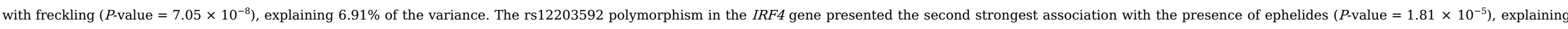

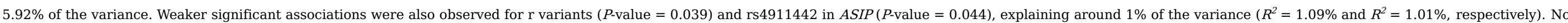
associations were found for the remaining six polymorphisms, although rs2153271 in BNC2 showed a marginal significant association with freckling $(P$-value $=0.067)$.

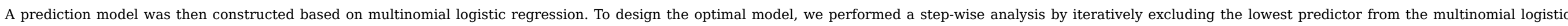

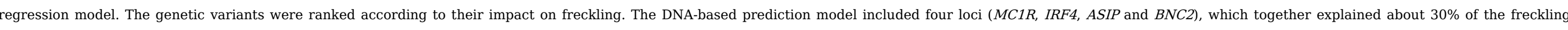

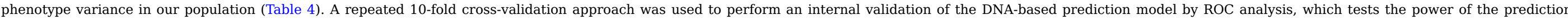
model [38]. The prediction accuracy of the tested DNA-based prediction model was $74.13 \%$ in the population, with an AUC of 0.771 , a specificity of $82.00 \%$, and a sensitivity of $63.51 \%$ (Fig. $1 \mathrm{~A}$ ).

Table 4 Multivariate logistic regression testing freckles association with genetic variants and sex.

\begin{tabular}{|c|c|c|c|c|c|c|}
\hline \multirow[b]{2}{*}{ Gene } & \multirow[b]{2}{*}{ SNP rs\# No. } & \multirow[b]{2}{*}{ Variable } & \multicolumn{2}{|c|}{ DNA-based prediction model } & \multicolumn{2}{|c|}{ Sex-adjusted prediction model } \\
\hline & & & OR $(95 \% \mathrm{CI})$ & $P$-value ${ }^{\mathrm{a}}$ & OR (95\% CI) & $P$-value ${ }^{\mathrm{a}}$ \\
\hline \multirow[t]{2}{*}{$M C 1 R$} & $\mathrm{R}$ alleles & $1 / 0$ & $4.18(2.49-7.02)$ & 6.66E-08 & $4.21(2.48-7.15)$ & 1.02E-07 \\
\hline & & $1 / 1$ & $21.51(4.50-102.79)$ & 1.20E-04 & $21.94(2.48-105.16)$ & 1.12E-04 \\
\hline \multirow[t]{2}{*}{ IRF4 } & rs12203592 & $\mathrm{CT}$ & $3.28(2.05-5.26)$ & 7.75E-07 & $3.26(2.02-5.27)$ & 1.43E-06 \\
\hline & & TT & $15.51(3.26-73.76)$ & $5.65 E-04$ & $14.63(3.06-70.10)$ & $7.81 \mathrm{E}-04$ \\
\hline \multirow[t]{2}{*}{$M C 1 R$} & $\mathrm{r}$ alleles & $1 / 0$ & $2.08(1.30-3.32)$ & 2.20E-03 & $2.05(1.28-3.30)$ & 2.92E-03 \\
\hline & & $1 / 1$ & $3.45(1.51-7.87)$ & 3.20E-03 & $3.39(1.43-7.99)$ & $5.35 \mathrm{E}-03$ \\
\hline
\end{tabular}




\begin{tabular}{|c|c|c|c|c|c|c|}
\hline \multirow[t]{2}{*}{$A S I P$} & rs4911442 & AG & $2.26(1.22-4.17)$ & $9.27 \mathrm{E}-03$ & $2.38(1.28-4.45)$ & $6.49 \mathrm{E}-03$ \\
\hline & & GG & $3.65(0.35-38.40)$ & 0.281 & $5.15(0.47-56.62)$ & 0.181 \\
\hline \multirow[t]{3}{*}{$B N C 2$} & rs2153271 & $\mathrm{CT}$ & $0.91(0.55-1.49)$ & 0.706 & $0.98(0.59-1.63)$ & 0.937 \\
\hline & & $\mathrm{CC}$ & $0.45(0.23-0.90)$ & 0.023 & $0.48(0.24-0.97)$ & 0.040 \\
\hline & & Male sex & - & - & $0.49(0.32-0.79)$ & 2.70E-03 \\
\hline
\end{tabular}

SNP, single nucleotide polymorphism; OR, odds ratio per minor allele; CI, confidence interval. Optimal model selection procedure was based on the Akaike information criterion (AIC).

a $P$-value for the multivariate logistic regression association analysis. Bold indicates significant results at Bonferroni threshold of $4.50 \mathrm{E}-03$. 


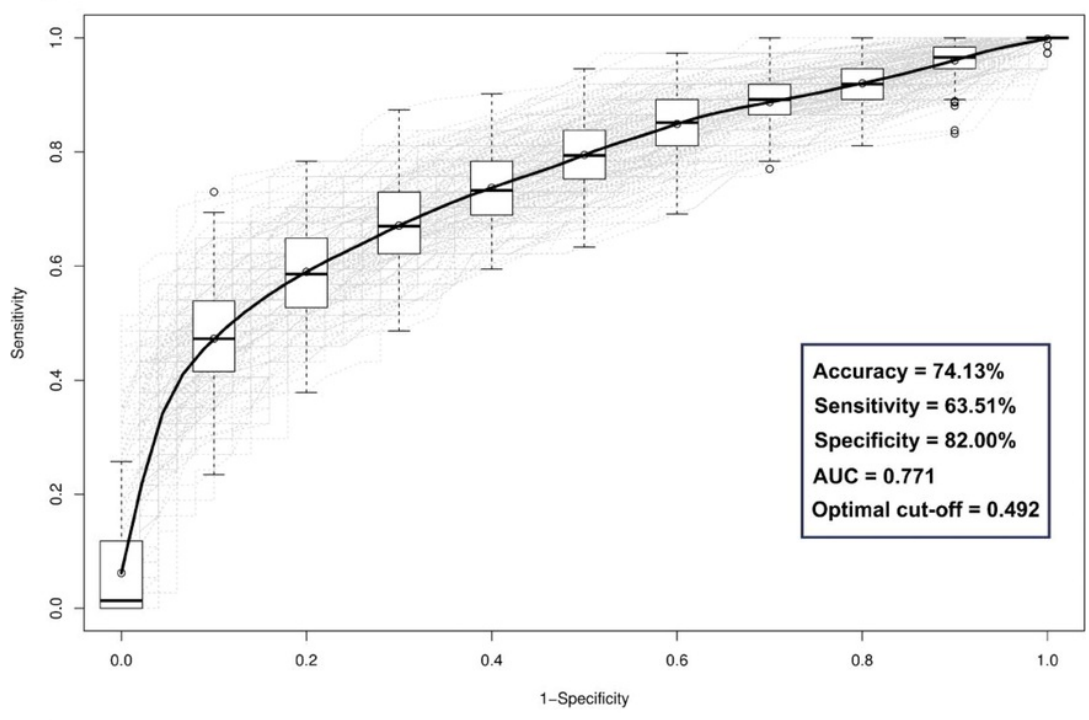

B)

Sex-adjusted prediction model

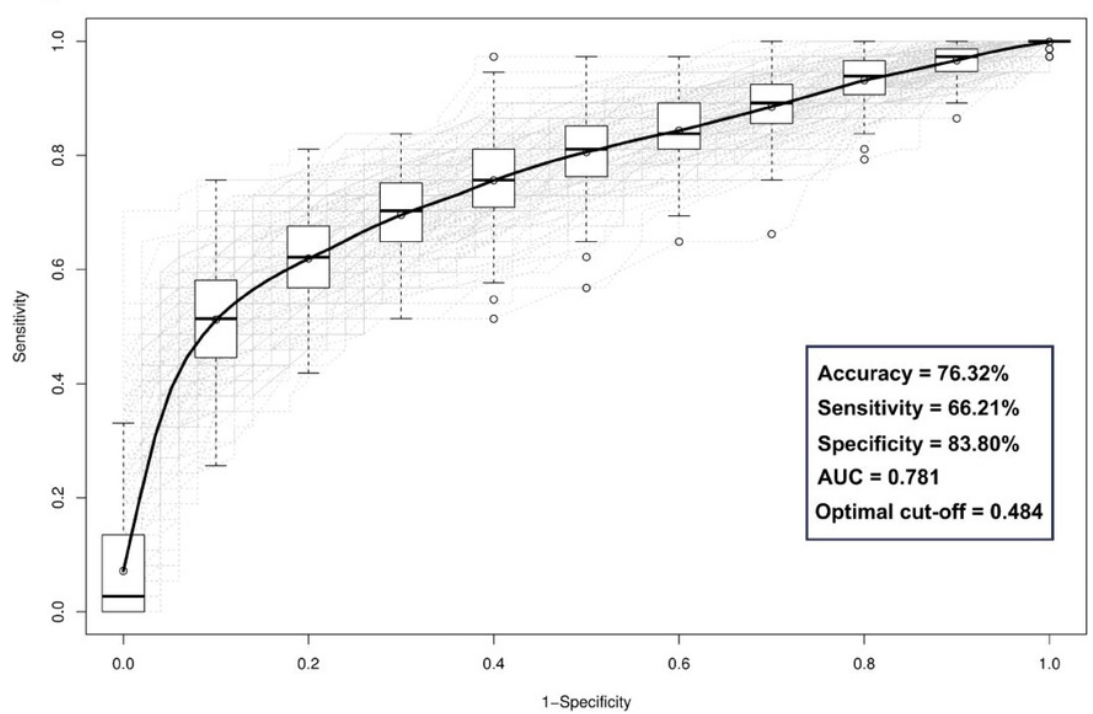

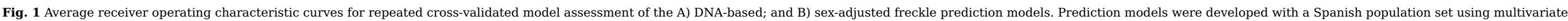

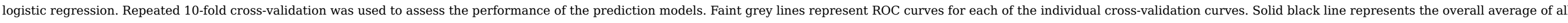
ROC curves. Mean values of the basic accuracy parameters are displayed. Optimal cut-off was selected for maximising Youden Index. AUC, area under the curve.

alt-text: Fig. 1

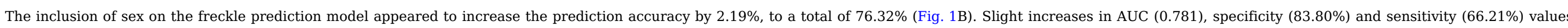

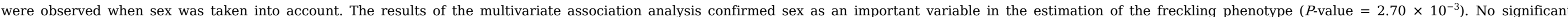




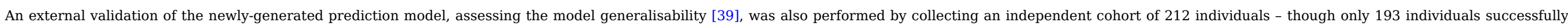

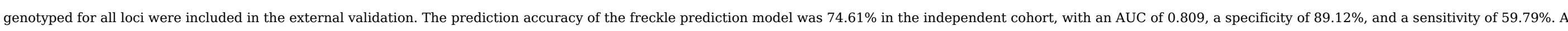
total of 49 individuals were incorrectly predicted (misclassification rate of 25.39\%) (Table 5).

Table 5 Accuracy of the sex-adjusted freckle prediction model obtained from an independent cohort of Spanish individuals.

alt-text: Table 5

\begin{tabular}{|c|c|c|c|c|c|c|c|c|}
\hline \multirow[t]{2}{*}{ Prediction model } & \multirow[t]{2}{*}{ Phenotype } & \multicolumn{2}{|c|}{ Predicted phenotype } & \multirow[t]{2}{*}{ Total $^{\mathrm{b}}$} & \multicolumn{4}{|c|}{ Prediction accuracy parameters } \\
\hline & & Freckled & Non-freckled & & AUC & Sensitivity (\%) & Specificity (\%) & Accuracy (\%) \\
\hline \multirow[t]{4}{*}{ Sex-based prediction modela } & Freckled & 55 & 37 & 92 & 0.809 & 59.79 & 89.12 & 74.61 \\
\hline & Non-freckled & 12 & 89 & 101 & & & & \\
\hline & Total & 67 & 126 & 193 & & & & \\
\hline & Fails (\%) & $12(17.91)$ & $37(29.36)$ & 49 (25.39) & & & & \\
\hline
\end{tabular}

a Optimal cut-off maximising Youden Index (0.6138) was used to classify individuals.

b Genetic analyses of four loci were successfully performed in 193 out of 212 individuals (missing rate of 8.96\%).

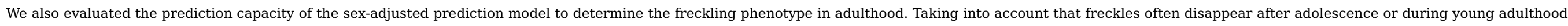

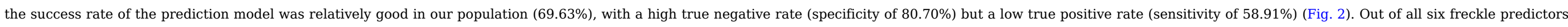
included in the model, only the r variants in $M C 1 R$ did not show a significant contribution to freckling in adulthood $(P$-value $>0.05)$.

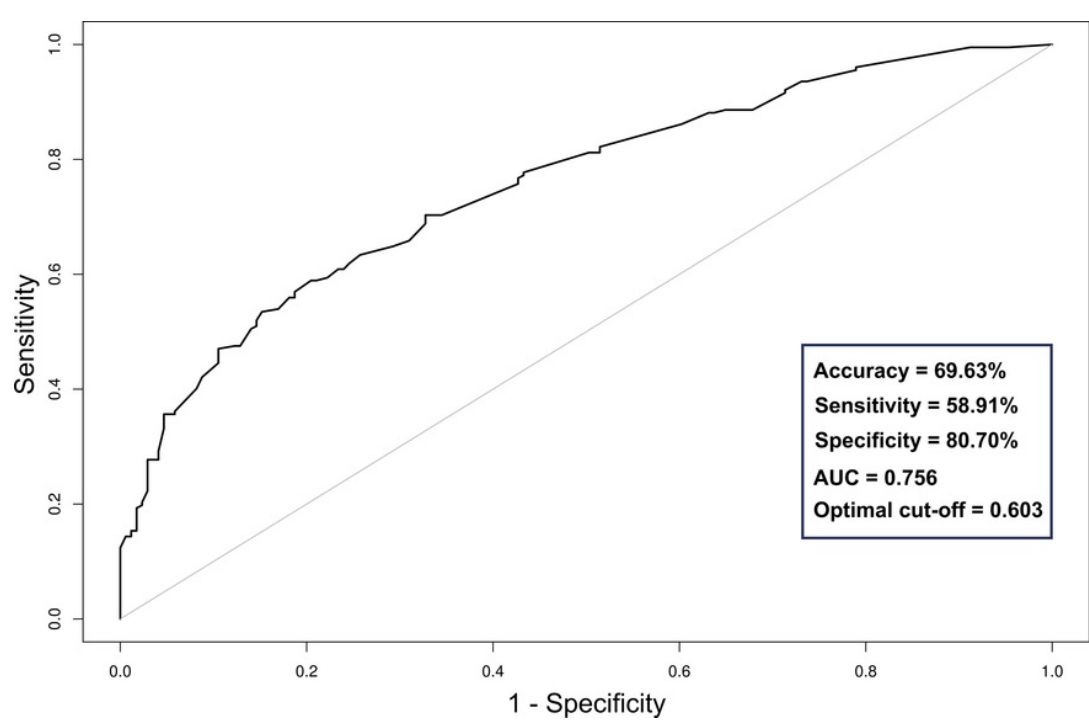

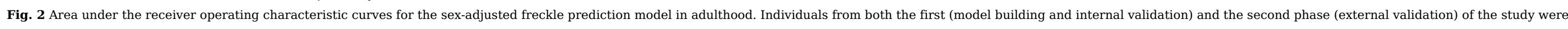
pooled to perform this analysis. Using Youden Index, optimal cut-off was selected for maximising the sensitivity and specificity of the prediction model to classify individuals. AUC, area under the curve. 


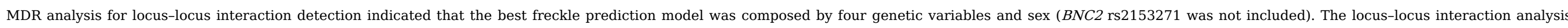

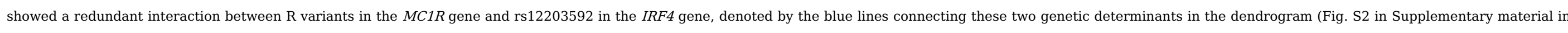

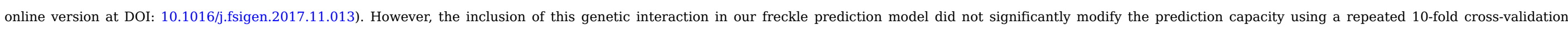
approach (AUC $=0.768$, specificity $=83.20 \%$, sensitivity $=61.27 \%$, and accuracy $=75.45 \%$ ).

\section{Discussion}

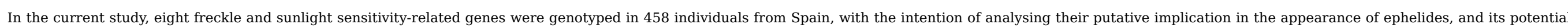

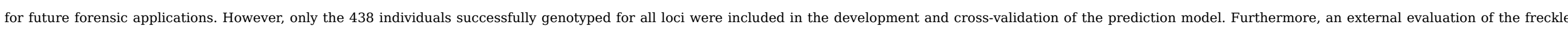
prediction model was performed on an extra 212 sample independent dataset by genotyping the genetic variants associated with freckling in the original population.

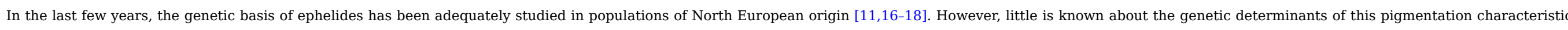

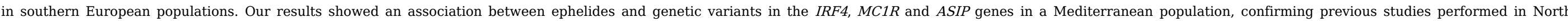

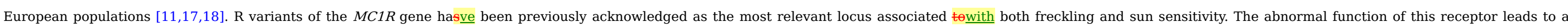

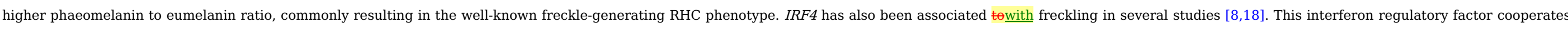

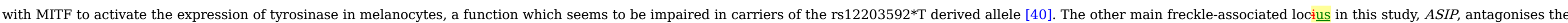

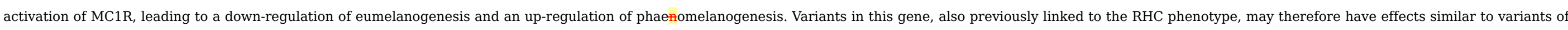
the $M C 1 R$ gene [16,17].

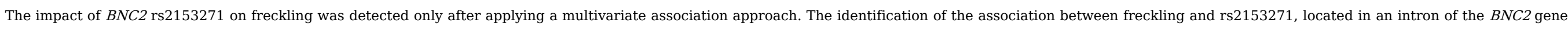
was recently discovered in a GWAS study performed in a population of North European ancestry [16], and was additionally correlated with acquired facial pigmented spots during aging [19].

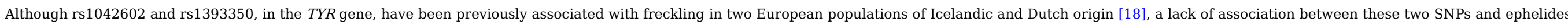

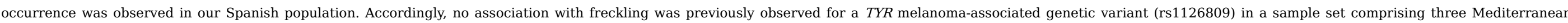

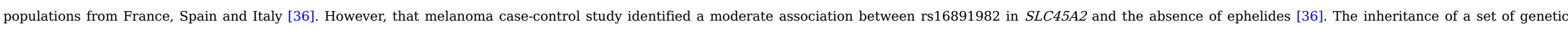

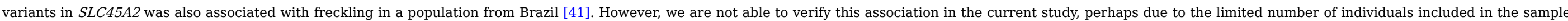
set.

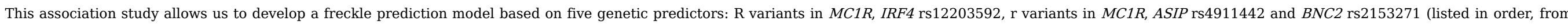

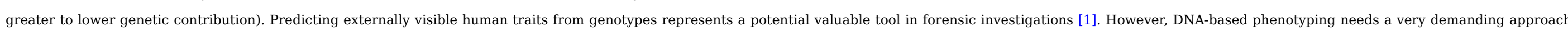

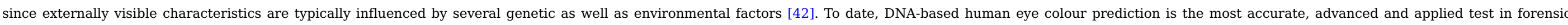

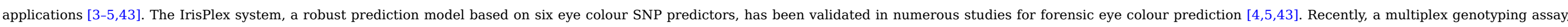

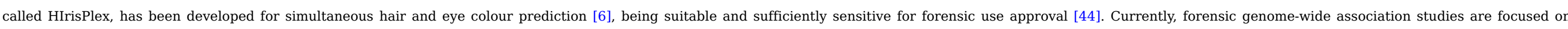

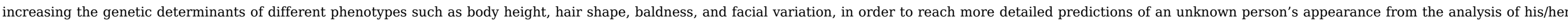
DNA [16,45-48].

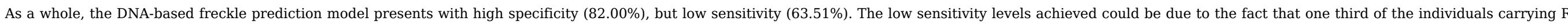

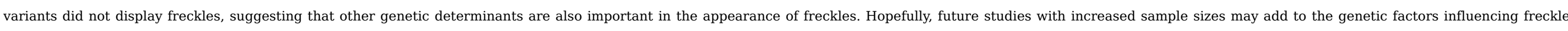
occurrence, so that the model's prediction potential may be significantly improved.

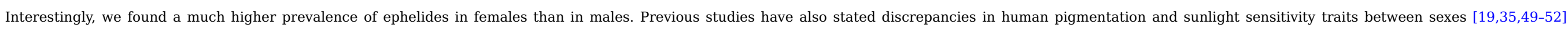

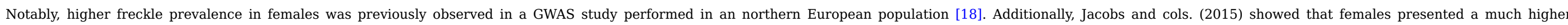
occurrence of facial sun spots than males, being the total variance explained by sex higher than any of the genetic variants studied [19]. 


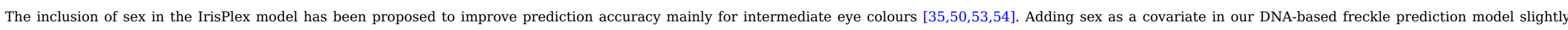

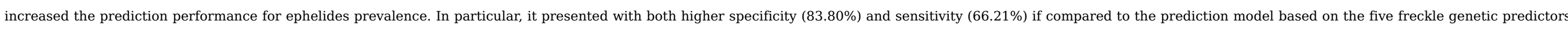

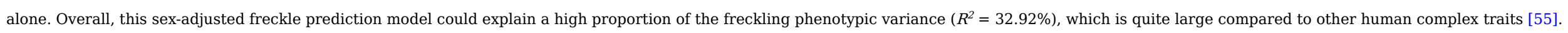

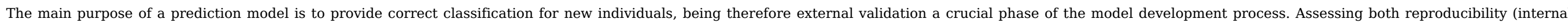

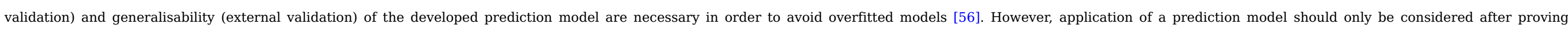

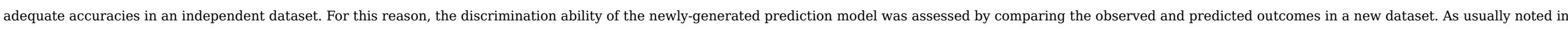
most external validations, a slight decrease of the overall prediction accuracy was observed (from $76.32 \%$ to $74.61 \%$ ).

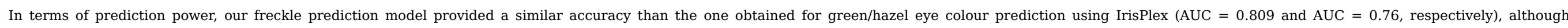

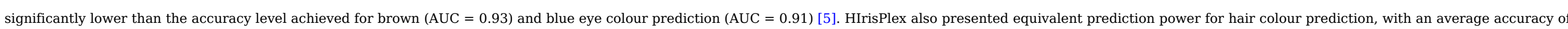

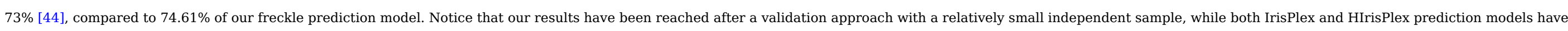
been validated in independent large population samples.

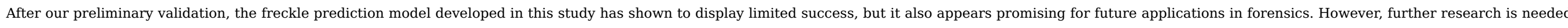
to increase the correct prediction rate (sensitivity) of the model, since the percentage of freckled individuals predicted as non-freckled is considerably high (false negative rate of $40.21 \%$ ).

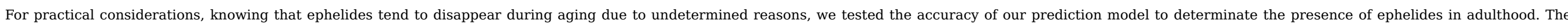

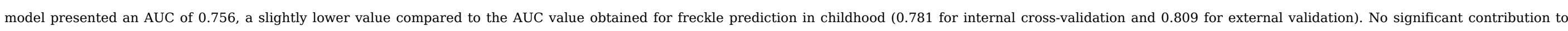

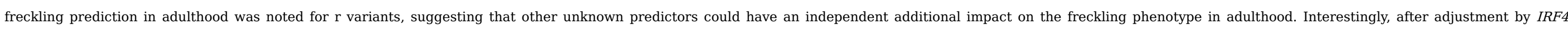

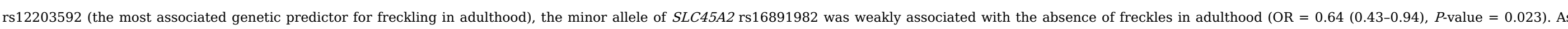

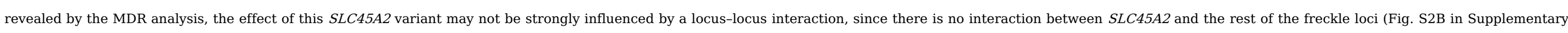
material in online version at DOI: 10.1016/j.fsigen.2017.11.013).

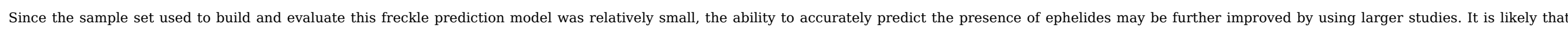

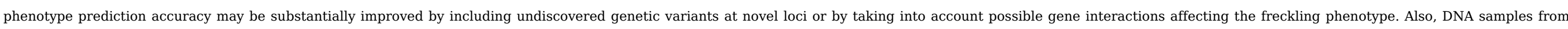
different populations of European origin should be included in further studies in order to validate this DNA predictive test for future forensic applications.

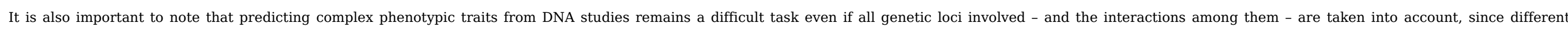
environmental factors may always have a considerable effect (for example, the effect of UV exposure on freckle occurrence).

\section{Conclusions}

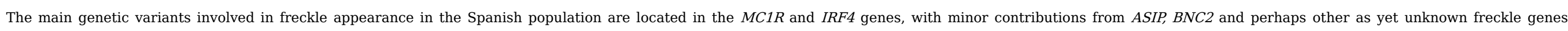

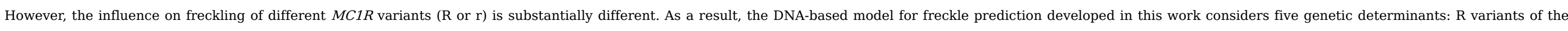

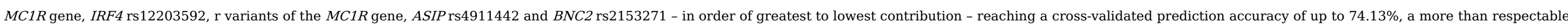
percentage. When sex is added to the model, the cross-validated prediction accuracy reached is even higher, growing up to $76.32 \%$.

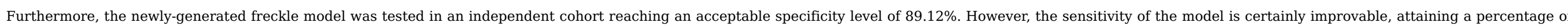
around $60 \%$.

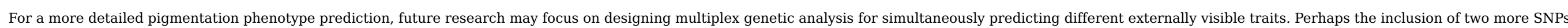

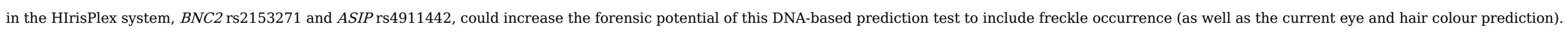

\section{Acknowledgements}




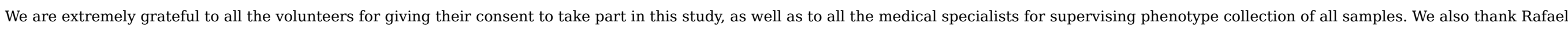
Velasco for providing us with the freckle photographs.

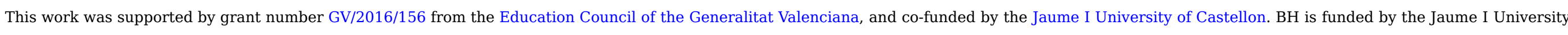
of Castellon under a Predoctoral Research contract (No. 15721).

\section{References}

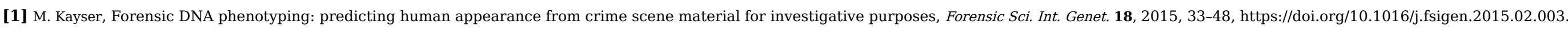

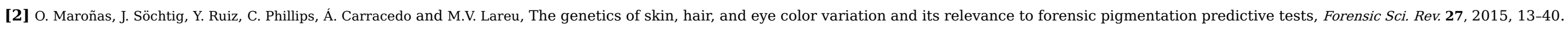

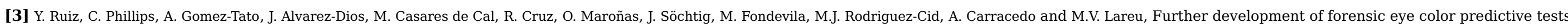
Forensic Sci. Int. Genet. 7, 2013, 28-40, https://doi.org/10.1016/j.fsigen.2012.05.009.

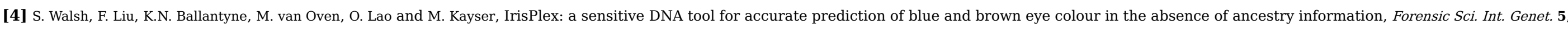
2011, 170-180, https://doi.org/10.1016/j.fsigen.2010.02.004

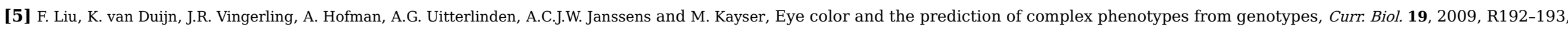
https://doi.org/10.1016/j.cub.2009.01.027.

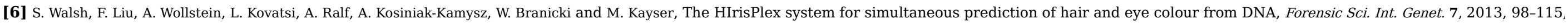
https://doi.org/10.1016/j.fsigen.2012.07.005.

[7] A. Pneuman, Z.M. Budimlija, T. Caragine, M. Prinz and E. Wurmbach, Verification of eye and skin color predictors in various populations, Leg. Med. Tokyo Jpn. 14, 2012, 78-83, https://doi.org/10.1016/j.legalmed.2011.12.005.

[8] C. Praetorius, R.A. Sturm and E. Steingrimsson, Sun-induced freckling: ephelides and solar lentigines, Pigment Cell Melanoma Res. 27, 2014, 339-350, https://doi.org/10.1111/pcmr.12232.

[9] V. Bataille, H. Snieder, A.J. MacGregor, P. Sasieni and T.D. Spector, Genetics of risk factors for melanoma: an adult twin study of nevi and freckles, J. Natl. Cancer Inst. 92, 2000 , 457-463.

[10] J.L. Rees, The genetics of sun sensitivity in humans, Am. J. Hum. Genet. 75, 2004, 739-751, https://doi.org/10.1086/425285.

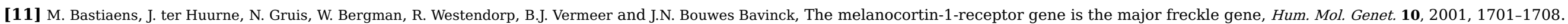

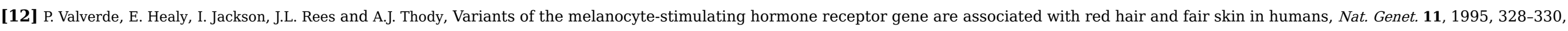
https://doi.org/10.1038/ng1195-328.

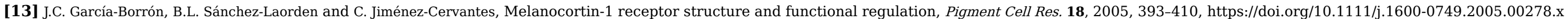

[14] J.V. Schaffer and J.L. Bolognia, The melanocortin-1 receptor: red hair and beyond, Arch. Dermatol. 137, 2001, 1477-1485.

[15] R.A. Sturm, R.D. Teasdale and N.F. Box, Human pigmentation genes: identification, structure and consequences of polymorphic variation, Gene 277, $2001,49-62$.

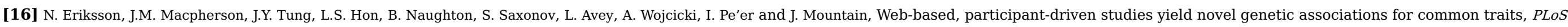
Genet. 6, 2010, e1000993, https://doi.org/10.1371/journal.pgen.1000993.

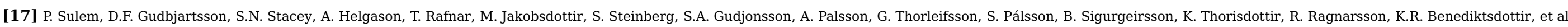
Two newly identified genetic determinants of pigmentation in Europeans, Nat. Genet. 40, 2008, 835-837, https://doi.org/10.1038/ng.160.

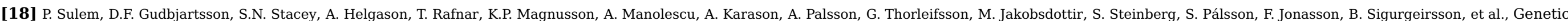




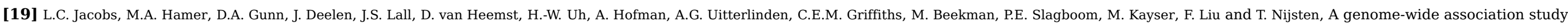
identifies the skin color genes IRF4, MC1R, ASIP, and BNC2 influencing facial pigmented spots, J. Invest. Dermatol. 135, 2015, 1735-1742, https://doi.org/10.1038/jid.2015.62.

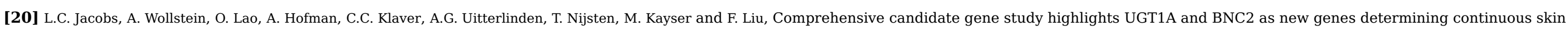
color variation in Europeans, Hum. Genet. 132, 2013, 147-158, https://doi.org/10.1007/s00439-012-1232-9.

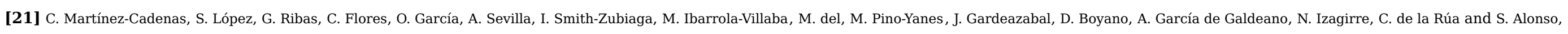
Simultaneous purifying selection on the ancestral MC1R allele and positive selection on the melanoma-risk allele V60L in south Europeans, Mol. Biol. Evol. 30, 2013, 2654-2665, https://doi.org/10.1093/molbev/mst158.

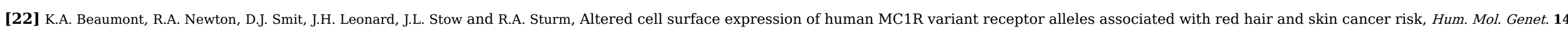
2005, 2145-2154, https://doi.org/10.1093/hmg/ddi219.

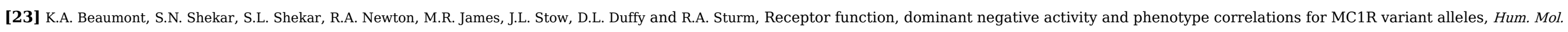
Genet. 16, 2007, 2249-2260, https://doi.org/10.1093/hmg/ddm177.

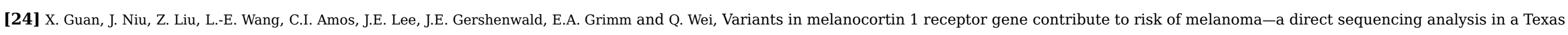
population, Pigment Cell Melanoma Res. 26, 2013, 422-425, https://doi.org/10.1111/pcmr.12070.

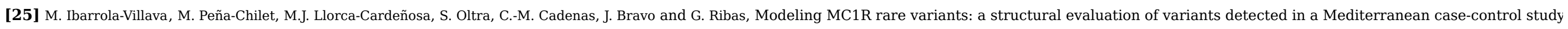
J. Invest. Dermatol. 134, 2014, 1146-1149, https://doi.org/10.1038/jid.2013.469.

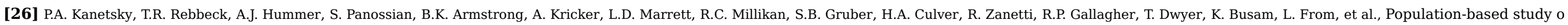
natural variation in the melanocortin-1 receptor gene and melanoma, Cancer Res. 66, 2006, 9330-9337, https://doi.org/10.1158/0008-5472.CAN-06-1634.

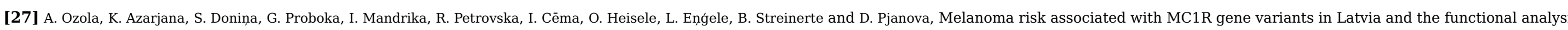
of rare variants, Cancer Genet. 206, 2013, 81-91, https://doi.org/10.1016/j.cancergen.2013.01.002.

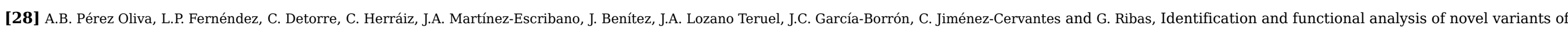
the human melanocortin 1 receptor found in melanoma patients, Hum. Mutat. 30, 2009, 811-822, https://doi.org/10.1002/humu.20971.

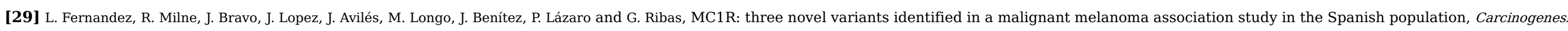
28, 2007, 1659-1664, https://doi.org/10.1093/carcin/bgm084.

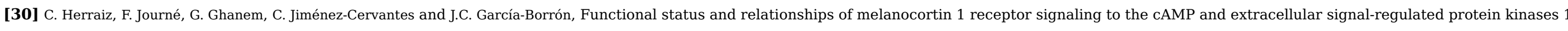
and 2 pathways in human melanoma cells, Int. J. Biochem. Cell Biol. 44, 2012, 2244-2252, https://doi.org/10.1016/j.biocel.2012.09.008.

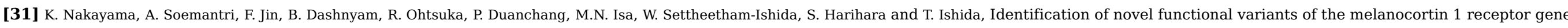
originated from Asians, Hum. Genet. 119, 2006, 322-330, https://doi.org/10.1007/s00439-006-0141-1.

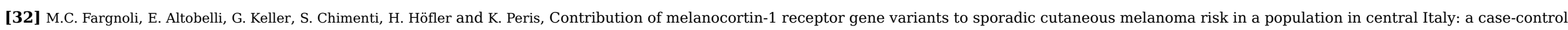
study, Melanoma Res. 16, 2006, 175-182, https://doi.org/10.1097/01.cmr.0000198454.11580.b5.

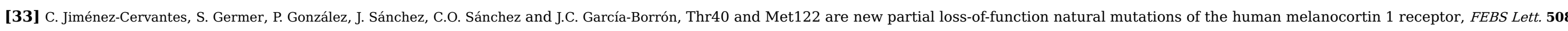
2001, 44-48.

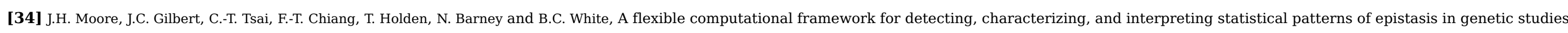


of human disease susceptibility, J. Theor. Biol. 241, 2006, 252-261, https://doi.org/10.1016/j.jtbi.2005.11.036.

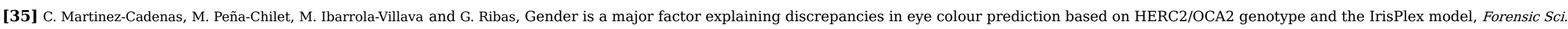
Int. Genet. 7, 2013, 453-460, https://doi.org/10.1016/j.fsigen.2013.03.007.

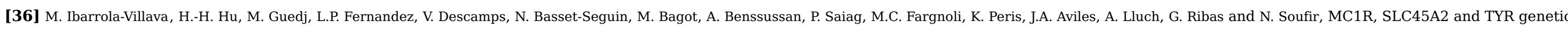

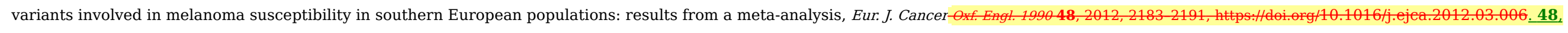
2012, 2183-2191.

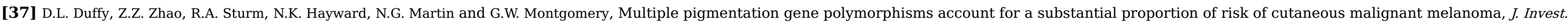
Dermatol. 130, 2010, 520-528, https://doi.org/10.1038/jid.2009.258.

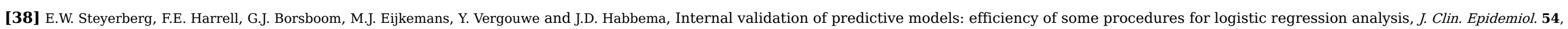
$2001,774-781$.

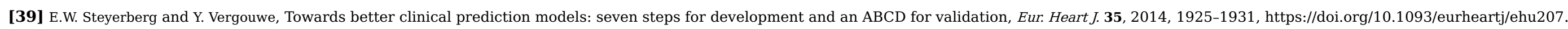

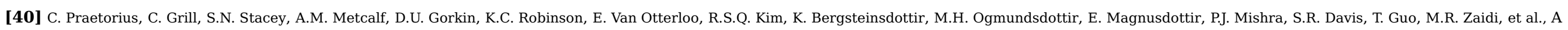
polymorphism in IRF4 affects human pigmentation through a tyrosinase-dependent MITF/TFAP2A pathway, Cell 155, 2013, https://doi.org/10.1016/j.cell.2013.10.022.

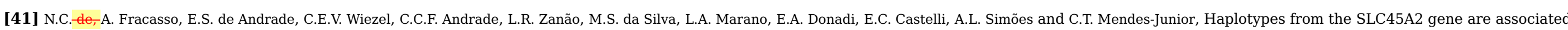
with the presence of freckles and eye, hair and skin pigmentation in Brazil, Leg. Med. 25, 2017, 43-51, https://doi.org/10.1016/j.legalmed.2016.12.013.

[42] F. Liu, B. Wen and M. Kayser, Colorful DNA polymorphisms in humans, Semin. Cell Dev. Biol. 24, 2013, 562-575, https://doi.org/10.1016/j.semcdb.2013.03.013.

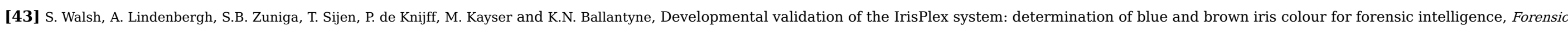
Sci. Int. Genet. 5, 2011, 464-471, https://doi.org/10.1016/j.fsigen.2010.09.008.

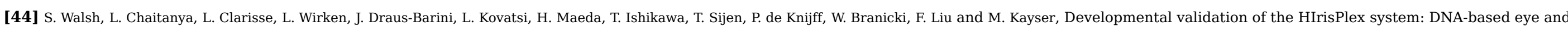
hair colour prediction for forensic and anthropological usage, Forensic Sci. Int. Genet. 9, 2014, 150-161, https://doi.org/10.1016/j.fsigen.2013.12.006.

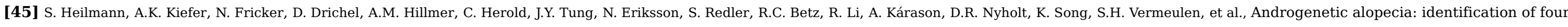
genetic risk loci and evidence for the contribution of WNT signaling to its etiology, J. Invest. Dermatol. 133, 2013, 1489-1496, https://doi.org/10.1038/jid.2013.43.

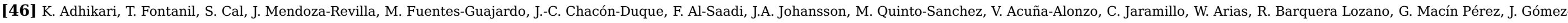

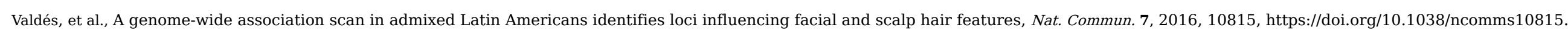

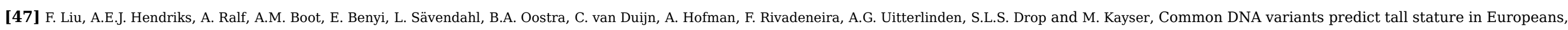
Hum. Genet. 133, 2014, 587-597, https://doi.org/10.1007/s00439-013-1394-0.

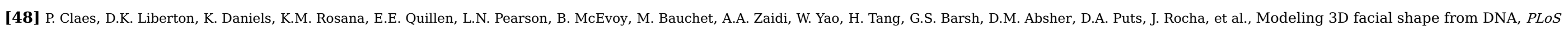
Genet. 10, 2014, e1004224, https://doi.org/10.1371/journal.pgen.1004224.

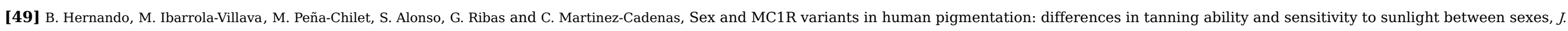
Dermatol. Sci. 84, 2016, 346-348, https://doi.org/10.1016/j.jdermsci.2016.09.004.

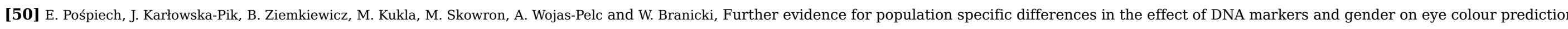
in forensics, Int. J. Legal Med. 130, 2016, 923-934, https://doi.org/10.1007/s00414-016-1388-2. 


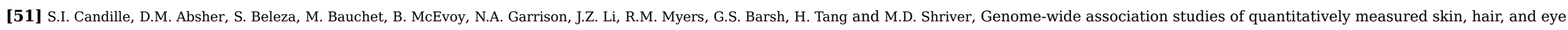
pigmentation in four European populations, PLoS One 7, 2012, e48294, https://doi.org/10.1371/journal.pone.0048294.

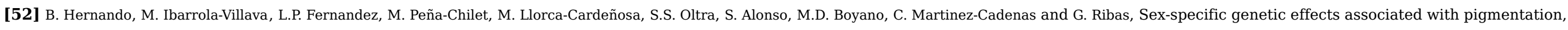
sensitivity to sunlight, and melanoma in a population of Spanish origin, Biol. Sex Differ. 7, 2016, 17, https://doi.org/10.1186/s13293-016-0070-1.

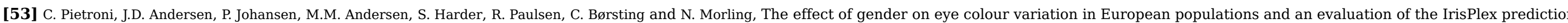
model, Forensic Sci. Int. Genet. 11, 2014, 1-6, https://doi.org/10.1016/j.fsigen.2014.02.002.

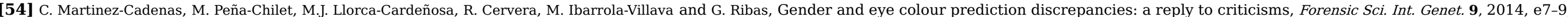
https://doi.org/10.1016/j.fsigen.2013.10.002

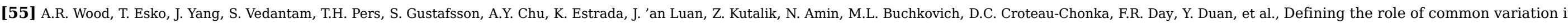
the genomic and biological architecture of adult human height, Nat. Genet. 46, 2014, 1173-1186, https://doi.org/10.1038/ng.3097.

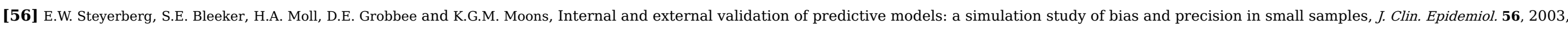

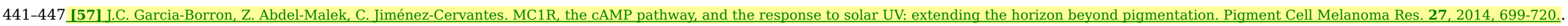

\section{$\nabla$ E-Extra}

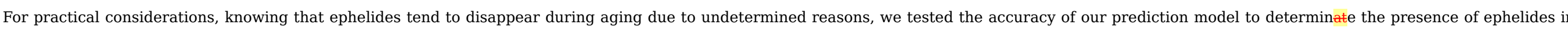

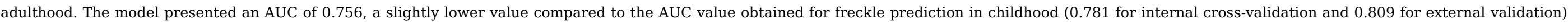

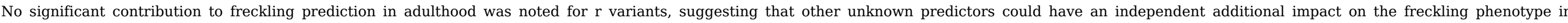

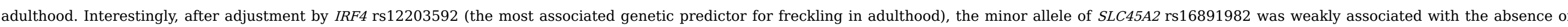

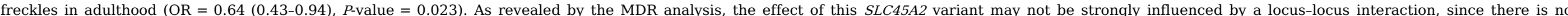
interaction between SLC45A2 and the rest of the freckle loci (Fig. S2B).

\section{$\nabla$ E-component}

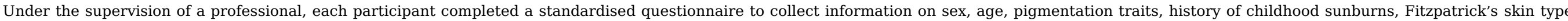

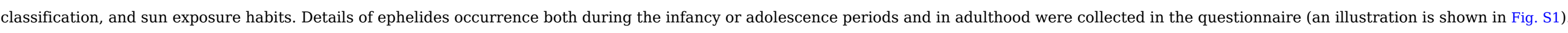
Multimedia Component 1

Fig. S1 Examples of heavily freckled, lightly freckled and non-freckled individuals of Mediterranean origin.

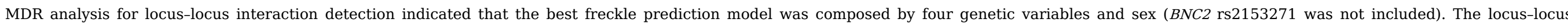

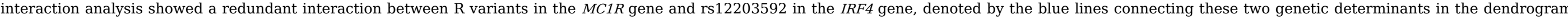

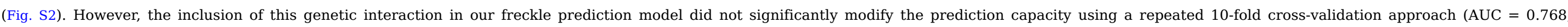
specificity $=83.20 \%$, sensitivity $=61.27 \%$, and accuracy $=75.45 \%$ ).

Multimedia Component 2

Fig. S2 Analysis using MDR software of locus-locus interactions influencing the freckling phenotype in A) childhood and B) adulthood. Blue lines shown in entropic dendrograms represents redundant interactions.

\section{Highlights}

- Prediction of human appearance from DNA is a useful tool to identify unknown persons. 
- A preliminary DNA-based prediction model for the presence of ephelides is developed.

- Accuracy of the newly-generated freckle prediction model is reasonably high.

- Further research is needed before practical use in forensics of the newly-generated freckle model.

\section{Queries and Answers}

Query: The author names have been tagged as given names and surnames (surnames are highlighted in teal colour). Please confirm if they have been identified correctly.

Answer: Author names have been identified correctly

Query: Please check the hierarchy of section headings.

Answer: The hierarchy of the section headings has been checked and it is correct

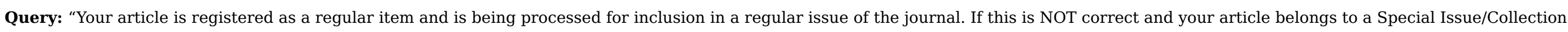
please contact a.healey@elsevier.com immediately prior to returning your corrections."

Answer: The article must be considered as a regular item, and should be included in a regular issue of the journal

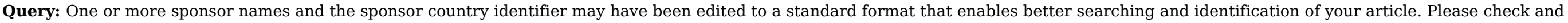
correct if necessary.

Answer: The name of the sponsor institution is adequate and correct. No need of further editing

Query: Please check the presentation of tables and correct if necessary.

Answer: Table presentation has been checked. No need for corrections

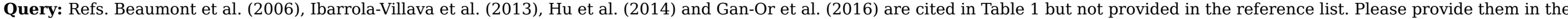
reference list or delete these citations from the table.

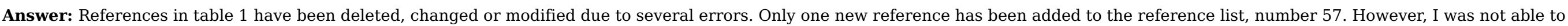

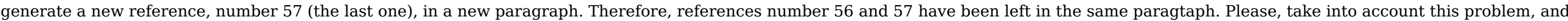
separate both references in two different paragraphs. 